\title{
Finite Element Simulation on Process Chain in Welding and Bending
}

\author{
Yuanbin FANG ${ }^{1,2, a}$, Can WANG ${ }^{1,2, b}$, Liping ZHANG ${ }^{1,2, c}$ \\ ${ }^{1}$ Jiangsu Xuzhou Engineering Machinery Research Institute, Jiangsu Xuzhou 221004, China \\ ${ }^{2}$ State key Laboratory of Intelligent Manufacturing of Advanced Construction Machinery, XCMG \\ Construction Machinery Co., Ltd, Jiangsu Xuzhou 221004, China \\ afybflying@163.com, ${ }^{b} 18251591969 @ 163 . c o m,{ }^{c} 15094347413 @ 163 . c o m$
}

\section{Keywords: Bending; welding; process chain; deformation}

Abstract. It simulates boom process chain in bending and welding. It obtains deformation of the plate after bending, and predicts welding deformation of the boom considering bending. At the same time, using Metra SCAN, finite element model is verified correctly by experiments. The results show that it occurs $4.3 \mathrm{~mm}$ deviation in the $90^{\circ}$ bending angle near the middle position, and $4.8 \mathrm{~mm}$ deviation in the $110^{\circ}$ bending angle. It is consistent in deformation trend, and proves favorably correctness of finite element model of bending. Deformation trend is contracted inwards. Peak value is located at the free end of weld position, and is $1.66 \mathrm{~mm}$. It is $1.9 \mathrm{~mm}$ deviation in the side of boom. The deviation is mainly located in the middle position of $90^{\circ}$ bending angle. The error between simulation results and experimental results are within $3 \mathrm{~mm}$. It meets the requirement of engineering application. It is consistent in deformation trend. It proves accuracy of finite element model of process chain in bending and welding.

\section{Introduction}

Welding deformation is a common problem, and harmful deformation of large structure is a waste of production [1]. In the past ten years, computer aided welding technology has been very mature. The contradiction in production and application is mainly embodied in calculation precision and calculation efficiency. But high strength steel is relatively thin, and the number of weld seams is small. Influence of structure deformation caused by quality of tailor welded. The problem is how to improve calculation accuracy of large-scale structure. It needs to be considered of before process. In the deformation control of box girder structure, there are two factors that are quality of bending precision and welding heat input. It is very important for optimizing process how to effectively predict process chain deformation of bending and welding.

Experts and scholars have done a lot of work on theoretical research of bending and welding process[2]. Murakawa, professor of Osaka University, used inherent strain theory to predict deformation of sheet metal [3]. J.H. Mo, professor of Huazhong University of Science and Techonogy,established finite element model of big-size U-style section through adjusting of bending paremeters[4]. G. Alexander, professor of Linköping University, used of finite element method to simulate process chain in stamping, spot welding of sheet metal and assembly sequence, and seted up a virtual prototype to predict deformation of the car after assembly[5]. In view of above research, they are more in-depth research in their respective areas. However, it is less for the coupled bending-welding of medium plate, which has a certain impact on accuracy of finite element model.

In this research, box girder structure is used as the object. It simulates and verifies forming process of the plate of boom through finite element analysis. Then they make bending deformation results as initial conditions. Based on thermal elastic-plastic, it simulates welding process of the boom, and obtains the distribution of welding deformation. It establishes finite element modeling of process chain in bending and welding. 


\section{Test preparation}

The boom is bent two times. It adopts dynamic display algorithm. Friction coefficient is set to 0.3. Forming speed is $3 \mathrm{~mm} / \mathrm{s}$, and forming time is $10 \mathrm{~s}$. Punch is down to bend the plate. Bending angle is $90^{\circ}$ and $110^{\circ}$. Respectively, the corresponding bending radius is $8 \mathrm{~mm}$ and $10 \mathrm{~mm}$. It is shown in Figure 1 (a). Boom length is $2980 \mathrm{~mm}$, and width is $190 \mathrm{~mm}$. Height is about $450 \mathrm{~mm}$ along the symmetry axis, and plate thickness is $5 \mathrm{~mm}$. Geometry of the boom is shown in Figure 1 (b).It is welded by Fronius 500 welding machine, and welding wire is ER50-6. The diameter of welding wire is $1.2 \mathrm{~mm}$, and the groove angle is $67^{\circ}$. Welding parameters are shown in table 1.

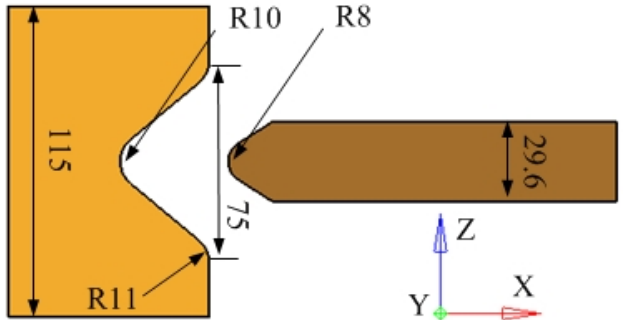

(a)The bending mould

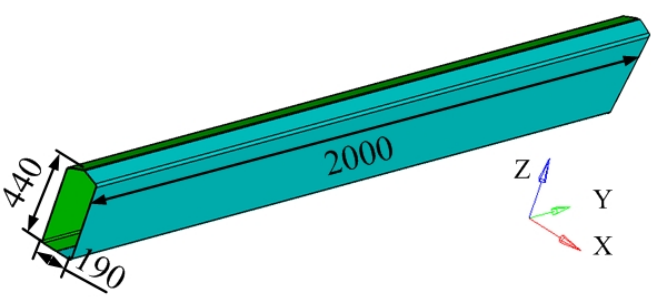

(b)Geometry of the boom

Fig. 1 The bending mould and geometry of the boom

\begin{tabular}{c|c|c}
\multicolumn{3}{c}{ Table 1 Welding parameters } \\
\hline Layers & The first layer & The second layer \\
\hline Arc voltage [V] & $22-23$ & $24-25$ \\
\hline Arc current [A] & $250-260$ & $240-260$ \\
\hline Welding speed [mm/s] & 42 & 12
\end{tabular}

Material of boom is Q460D, and varies with temperature. It needs to be considered thermal physical and mechanical parameters of material of in welding simulation. It selects ambient temperature as material mechanical property parameters in bending simulation. Physical parameters and mechanical parameters change with temperature. It is shown in Figure 2. Material density is $7860 \mathrm{~kg} / \mathrm{m}^{3}$, and elongation is least $16 \%$.

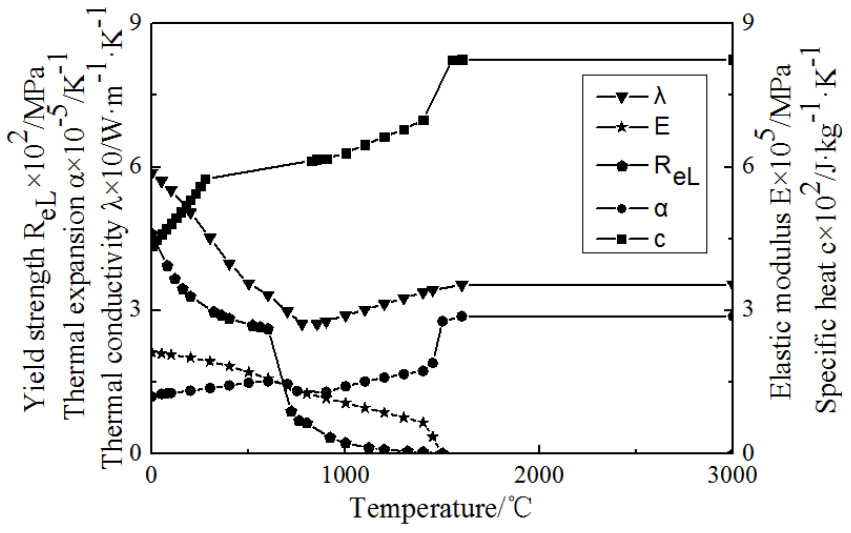

Fig. 2 Relationship between thermo-mechanical parameters and temperature of Q460D

\section{Establishment of finite element model}

In order to effectively control model calculation and bending precision, it deals with dense grid in the bending positions, weld seams and heat-affected zone. It adopts large grid away from weld seam positions. Mesh model of the plate and boom is shown in Fig. 3. The minimum size of grid is $1 \mathrm{~mm}$, and the maximum size of grid is $54 \mathrm{~mm}$. The total number of grid unit is 61716 , and the number of node is 93906. 


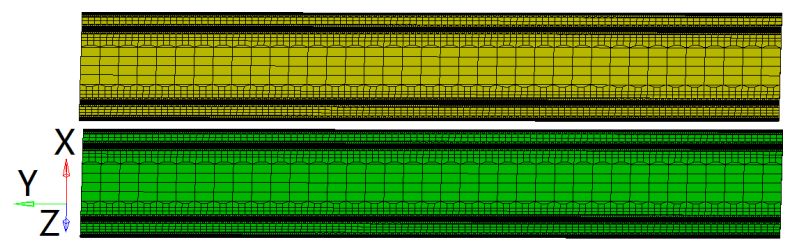

(a)Mesh model of the bending mold

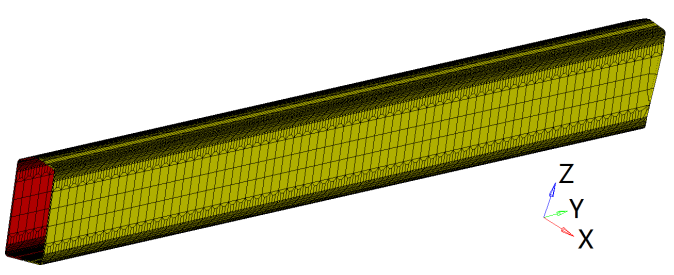

(b)Mesh model of the boom

Fig. 3 Finite element model

Ignoring the flow of welding molten pool, it is defined as quasi steady state. Establish the proper heat source model reflects carbon-dioxide arc welding [6]. Double ellipsoid heat source fully considers the characteristic of heat source that temperature of front heat source changes steeply, and temperature of rear heat source changes slowly in the welding process.

Cumulate distribution function of front-end and rear-end ellipsoid heat source is showed in Eq. 1 and Eq. 2.

$$
\begin{aligned}
& q(x, y, z, t)=\frac{6 \sqrt{3} 0 f_{1}}{a_{1} b c \pi \sqrt{\pi}} \exp \left\{-3\left[\frac{x^{2}}{c^{2}}+\frac{y^{2}}{b^{2}}+\frac{(z-v t)^{2}}{a_{1}^{2}}\right]\right\} . \\
& q(x, y, z, t)=\frac{6 \sqrt{3} 0 f_{2}}{a_{2} b c \pi \sqrt{\pi}} \exp \left\{-3\left[\frac{x^{2}}{c^{2}}+\frac{y^{2}}{b^{2}}+\frac{(z-v t)^{2}}{a_{2}^{2}}\right]\right\} .
\end{aligned}
$$

where F1, F2 definite heat flow density distribution coefficient. Q definite input heat power, V definite welding speed. The parameters of $a_{1}, a_{2}, b$ and $c$ definite shape of double ellipsoid heat resource. $a_{1}$ and $a_{2}$ definite respectively the length of first and second half of double ellipsoid heat resource. $c$ affects width of welding. $b$ affects penetration of welding.

It measures welding high, bead width, penetration depth and heat affected zone. It obtains macro morphology of test specimen. It is shown in figure 4 . It gets heat source parameters that $\mathrm{a}_{1}$ is $7 \mathrm{~mm}, \mathrm{a}_{2}$ is $17.5 \mathrm{~mm}, \mathrm{~b}$ is $8.4 \mathrm{~mm}, \mathrm{c}$ is $6.2 \mathrm{~mm}$. Weld prefabricated grids are filled. Elements based on life-death technique are used to activate. Using the Newton's law and Pohl Weitzman's law, it takes into account respectively of convection and radiation between the boom and external environment. The convection heat transfer coefficient is set to 0.02 , and ambient temperature is set at $20^{\circ} \mathrm{C}$.

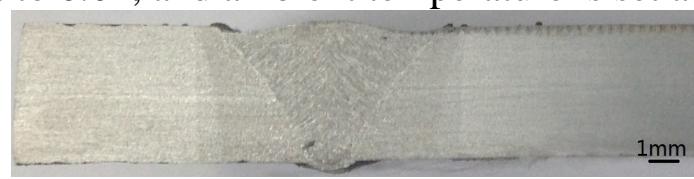

Fig. 4 Heat resource

The contact is adopted in the bending condition, and is not adopted in the rebound condition. The punch has a close velocity. Punch displacement is defined by displacement in Eq. 3.

$$
\mathrm{h}=\frac{1}{2} \cot \frac{\alpha}{2}-(\mathrm{r}+\mathrm{t}) \tan \frac{180^{\circ}-\alpha}{4} \cot \frac{\alpha}{2} \text {. }
$$

Where $h$ is punch displacement when punch contact with the plate, $\alpha$ is bending angle, $R$ is bending radius, $\mathrm{t}$ is plate thickness.

With bending, stress reaches yield limits away from the neutral layer. It occurs plastic deformation. Material near neutral layer is in the range of elastic deformation. When stress is eliminated, there will be elastic rebound. Rebound value is showed in Eq. 4.

$$
\beta=\alpha-\alpha_{\mathrm{n}}=\frac{3 \alpha \mathrm{r} \sigma_{\mathrm{s}}}{\mathrm{Et}} .
$$

Where $\beta$ is rebound value, $\alpha_{\mathrm{n}}$ is bending angle after rebound, $\sigma_{\mathrm{S}}$ is the yield strength, $\mathrm{E}$ is elastic modulus. 
The deformation of each node after the first $90^{\circ}$ bending is regarded as predeformation of the second bending. The influence of prior procedure on the current process is considered.

In order to avoid rigid-body displacement, it needs to definite equivalent boundary condition. When it welds the butt joint, it sets two groups of contact to definite the relation of tooling and boom. When it welds the $140^{\circ}$ joint, it sets one group of contact to definite the relation of tooling and boom. It is definition of constraints to restrict $\mathrm{Y}$ direction in the middle section to limit longitudinal contraction deformation. It is definition of constraints to restrict $\mathrm{X}$ displacement in the free section of boom to limit transverse shrinkage.

\section{Simulation results analysis and experimental verification}

Bending angle and welding deformation results analysis. It measures bending angle with angle measuring instrument. Because of the influence factors,such as moudle wear, uniformity and integrity of the plate in different manufacturers. They have an effect on bending. Through a series of experiments, they tests theoretical bending angle, punch deformation and measured bending angle. It obtains test results as shown in table 2 .

Tab. 2 Measurements of angle

\begin{tabular}{c|c|c|c|c|c|c}
\hline theoretical bending angle $[\mathrm{o}]$ & 90 & 87 & 84.5 & 110 & 104 & 97 \\
\hline Punch deformation[mm] & 21.75 & 28.40 & 29.48 & 19.47 & 21.38 & 23.76 \\
\hline measured bending angle[o] & 100 & 95 & 90 & 125 & 118 & 110
\end{tabular}

Table 3 data can be seen that punch deformation is $29.48 \mathrm{~mm}$ at the $90^{\circ}$ bending angle, and punch deformation is $23.76 \mathrm{~mm}$ at the $110^{\circ}$ bending angle.

It scans the shape of bent structure using Metra SCAN laser scanner. It records in the form of point cloud. At the same time, simulation results are compared with scanning results as shown in figure 5.

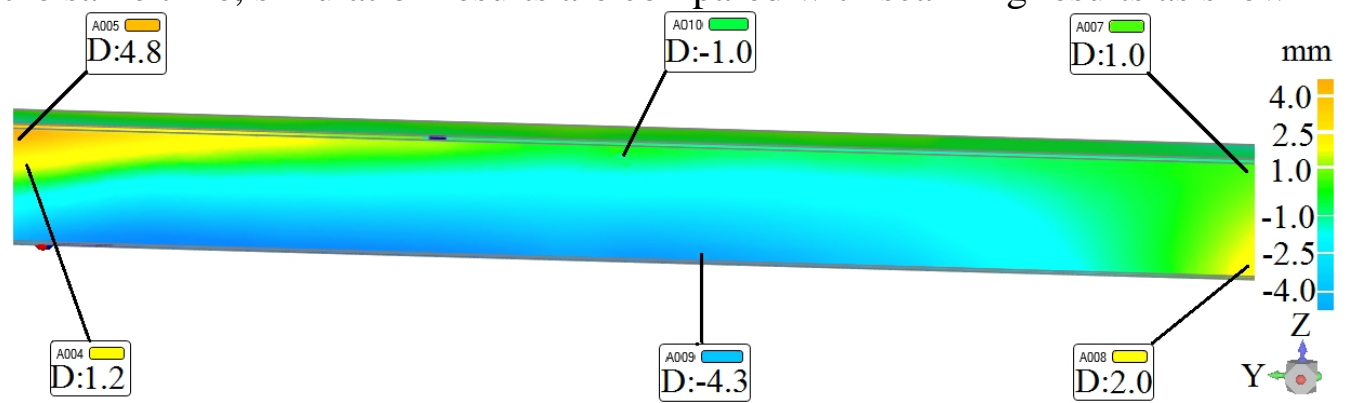

Fig.5 Compared with simulation and scanning results

It can be seen in Figure 5. It occurs $4.3 \mathrm{~mm}$ deviation in the $90^{\circ}$ bending angle near the middle position, and $4.8 \mathrm{~mm}$ deviation in the $110^{\circ}$ bending angle. It is consistent in deformation trend, and proves favorably correctness of finite element model of bending. The deviation of bending process is related to plate precision. Finite element model is established based on some assumptions, such as uniformity of material, flatness of plate and so on. According to comparison results, bending deviation position is used mechanical method to adjust.

Welding deformation analysis and test verification. Bending results are applied to boom welding process by predeformation. It obtains welding deformation as shown in figure 6 .

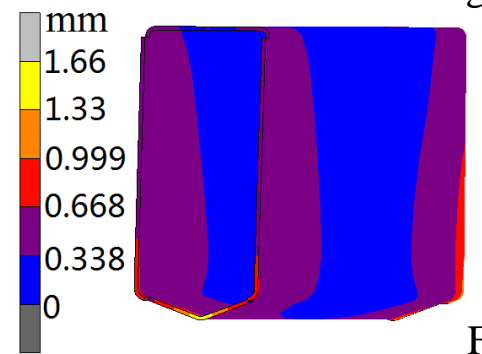

Fig.6 Contour of welding deformation

Deformation trend is contracted inwards. Peak value is located at the free end of weld position, and is $1.66 \mathrm{~mm}$. Welding deformation is upward in the weld seam position. The positions of arc striking and arc suppression are upward more obvious at the $140^{\circ}$ joint. Bending angle trend is larger. It dues to the two weld seams binding effect on shrinkage. 
In the same way, laser scanner scans shape of the boom. By least square method, it analyzes automatically test and simulation results with the software of geomagic qualify. The blue shows scan results, and dark green shows simulation results. It analyzes the compared results and marks error positions as shown in figure 7.

Figure 7 shows visually that it is $1.9 \mathrm{~mm}$ deviation in the side of boom. The deviation is mainly located in the middle position of $90^{\circ}$ bending angle. It uses least square method to solve model matching data, and the deviation is uniform distribution. It has cumulative error caused by certain bending. According to deviation values of deformation, error between simulation results and experimental results are within $3 \mathrm{~mm}$. It meets the requirement of engineering application. It is consistent in deformation trend. It proves accuracy of finite element model of process chain in bending and welding.

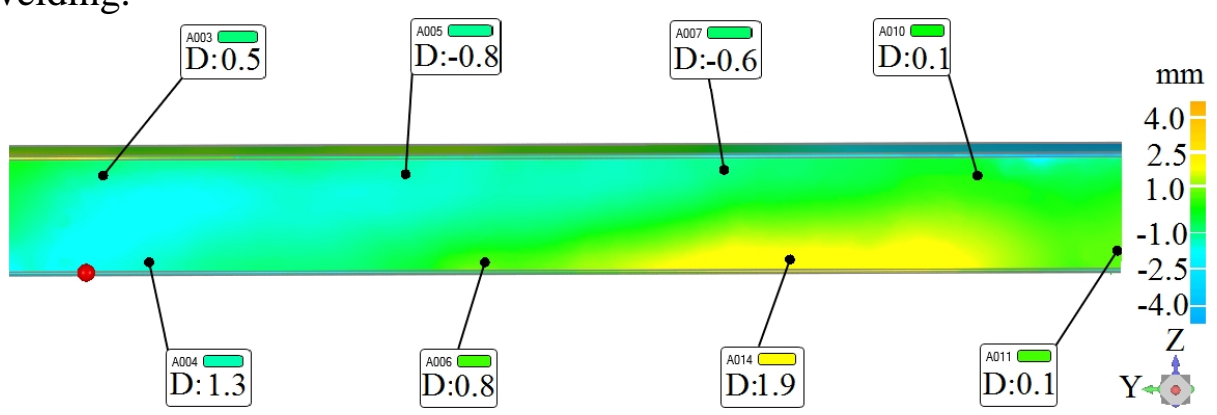

Fig.7 Compared with simulation and scanning results

\section{Conclusions}

It carries out numerical simulation of process chain in bending and welding. In aid of Metra SCAN laser scanner, it records profile of the plate, and verifies bending precision. Bending deformation is used as predeformation for welding simulation. It predicts welding deformation and verifies by experiments. The following conclusions are drawn in the research:

(1) There is a certain deviation in the middle position of $90^{\circ}$ bending angle and the front position of $110^{\circ}$ bending angle. Deformation trend of simulation and experimental results is consistent, and proves favorably correctness of finite element model of bending.

(2) Deformation trend is contracted inwards. Peak value is located at the free end of weld position.

(3) There is a large deviation in the side of boom. According to deviation values of deformation, error between simulation results and experimental results meets the requirement of engineering application. It is consistent in deformation trend. It proves accuracy of finite element model of process chain in bending and welding.

This study has not been able to carry out detailed analysis of bending adjustment process, which may lead to inconsistency of bending plates. The model can be further corrected by increasing process control and experiments, so that finite element model can be further optimized.

\section{Acknowledgements}

This work was financially supported by the Jiangsu Natural Science Foundation (BK20140229), and National Science and Technology Support Program (2015BAF07B02).

\section{References}

[1] X. T. Tian: Welding Structure (China Machine Press Publications, China 1982).

[2] K. Thomas, E. Andrea, H. André and L. Tobias: Key Engineering Materials, Vol. 651-653 (2015), p. 1312-1318.

[3] D. Deng, H. Murakawa and N. Ma: Science and Technology of Welding and Joining, Vol. 17, No. 1 (2012), p. 13-21. 
[4] X. L. ZHAO and J. H. Mo: China Metal Forming Equipment \& Manufacturing Technology, Vol. 17, No. 1 (2012), p. 13-21.

[5] G. Alexander, N. Larsgunnar and M. Ramin: Journal of Materials Processing Technology, Vol. 212, No. 7 (2012), p. 1453-1462.

[6] Y. B. Fang, X. M. Zong, H. Q. Zhang, B. Sun and X. Q. Yin: Welding Technology, Vol. 45, No. 3 (2016), p. 5-8. 